\title{
Mutations in the maternally imprinted gene MKRN3 are common in familial central precocious puberty
}

\author{
Dominique Simon ${ }^{1,2,3}$, Ibrahima Ba4, Nancy Mekhail' ${ }^{1}$ Emmanuel Ecosse', \\ Anne Paulsen ${ }^{1}$, Delphine Zenaty ${ }^{1,2,3}$, Muriel Houang ${ }^{5}$, Monique Jesuran \\ Perelroizen $^{6}$, Gian-Paolo de Filippo ${ }^{7}$, Mariacarolina Salerno ${ }^{8}$, Gilbert Simonin ${ }^{\text {, }}$ \\ Rachel Reynaud ${ }^{9}$, Jean-Claude Carel ${ }^{1,2,3}$, Juliane Léger ${ }^{1,2,3}$ and Nicolas de Roux ${ }^{2,3,4}$ \\ ${ }^{1}$ AP-HP, Service d'Endocrinologie Diabétologie Pédiatrique, Centre de Référence des Maladies Endocriniennes Rares \\ de la Croissance, Hôpital Robert Debré, Paris F-75019, France, ${ }^{2}$ Université Paris Diderot, Sorbonne Paris Cité F-75019, \\ France, ${ }^{3}$ Institut National de la Santé et de la Recherche Médicale (INSERM), Unité 1141, DHU Protect, Paris F-75019, \\ France, ${ }^{4}$ AP-HP, INSERM U1141, Laboratoire de Biochimie, Hôpital Robert Debré, 48 Boulevard Sérurier, Paris \\ F-75019, France, ${ }^{5}$ AP-HP, Explorations Fonctionnelles Endocriniennes, Hôpital Armand Trousseau, Paris F-75012, \\ France, ${ }^{6}$ Centre d'Endocrinologie Pédiatrique, 14 Rue du Rempart St-Etienne, Toulouse F-31000, France, ${ }^{7}$ Pediatric \\ Endocrinology Unit, Gaetano Rummo Hospital, Benevento 82100, Italy, ${ }^{8}$ Pediatric Endocrinology Unit, Federico II \\ University, Naples 80131, Italy and ${ }^{9}$ Service de Pédiatrie Multidisciplinaire, Centre de Référence des Maladies Rares \\ d'Origine Hypophysaire, Assistance Publique-Hopitaux de Marseille (APHM), Hôpital de la Timone, Aix-Marseille \\ Université, Marseille F-13385, France
}

\author{
Correspondence \\ should be addressed \\ to $\mathrm{N}$ de Roux \\ Email \\ nicolas.deroux@inserm.fr
}

\begin{abstract}
Context and objective: Idiopathic central precocious puberty (iCPP) is defined as early activation of the hypothalamicpituitary-gonadal axis in the absence of identifiable central lesions. Mutations of the makorin RING finger 3 (MKRN3) gene are associated with iCPP. We aimed to assess the frequency of MKRN3 mutations in iCPP and to compare the phenotypes of patients with and without MKRN3 mutations.

Design: An observational study was carried out on patients recruited at pediatric hospitals in France and Italy. Forty-six index CPP cases were screened for mutations in the MKRN3 coding sequence: 28 index cases of familial cases and 18 cases did not report any familial history of CPP. The endocrine phenotype was compared between MKRN3 mutated and non-mutated patients.
\end{abstract}

Results: MKRN3 mutations were identified in one sporadic and 13 familial cases. We identified five new heterozygous missense mutations predicted to be deleterious for protein function and two frameshift mutations, one new and the other recurrent, predicted to result in truncated proteins. Age at puberty onset varied very little among patients with $M K R N 3$ mutations and puberty occurred earlier in these patients than in those without MKRN3 mutations (6.0 years (5.4-6.0) vs 7.0 years (6.0-7.0), $P=0.01$ ).

Conclusions: MKRN3 mutations are common in familial iCPP. MKRN3 is one of the gatekeepers of the postnatal activation of the gonadotropic axis.

\section{Introduction}

Precocious puberty is usually defined as breast (B) development (Tanner B2) before 8 years of age in girls and gonad (G) development (Tanner G2, volume $>3 \mathrm{ml}$ or length $>25 \mathrm{~mm}$ ) before 9.5 years of age in boys (1).
Central precocious puberty (CPP) results from early activation of the hypothalamic-pituitary-gonadal axis. CPP can be due to hypothalamic lesions (2). CPP has also been reported in patients with genetic defects, such as (c) 2016 European Society of Endocrinology Printed in Great Britain
Published by Bioscientifica Ltd. 
maternal unidisomy of chromosome 14 (3), the dup (18q) and del (11q) syndromes (4), and Williams-Beuren syndrome (5). However, CPP is usually idiopathic CPP (iCPP). de Vries et al. (6) reported that about one-third of iCPP cases were familial. Segregation analysis for the affected families suggested an autosomal mode of inheritance, with incomplete sex-dependent penetrance.

Mutations or SNPs associated with CPP were initially described in $\operatorname{KISS} 1$ and $\operatorname{KISS} 1 R(7,8,9)$, but mutations of these genes are very rare in sporadic cases of iCPP (10). A recent whole-exome sequencing study identified mutations in the makorin RING finger 3 (MKRN3) gene in 15 individuals with iCPP (11). The mutation was inherited from the father in all 15 cases, consistent with the maternal imprinting of $M K R N 3$. Other loss-of-function $M K R N 3$ mutations have since been reported $(12,13,14,15)$. $M K R N 3$, located on chromosome 15q11.2, in the critical region for Prader-Willi syndrome, encodes MKRN3 protein. Most patients with Prader-Willi syndrome display hypogonadotropic hypogonadism, but iCPP has also been reported in a very small number of patients (16). The mechanism by which a loss of MKRN3 function leads to early activation of the hypothalamic-pituitary axis is unknown, although it has been suggested that MKRN3 inhibits the neuronal network controlling the secretion of gonadotropin-releasing hormone (GnRH) (11).

In this study, we investigated the frequency of $M K R N 3$ mutations in a large group of patients with iCPP, with the aim of describing the phenotype of patients carrying MKRN3 mutations, and to compare this phenotype with that of patients having iCPP but no identifiable MKRN3 mutations.

\section{Subjects and methods}

\section{Patients}

This analysis is a retrospective analysis of the endocrine phenotype of 46 index cases ( 45 girls and one boy) who have been screened for MKRN3 mutations. They were originated from France (44) and Italy (2). They were all negative for a mutation in KISS1 and KISS1R coding sequences. All cases had iCPP, defined as Tanner stage 2 (B2 or G2) before 8 years of age in girls and 9.5 years in boys, basal and/or GnRH-stimulated luteinizing hormone (LH) levels within the pubertal range, as defined in the following section, and normal findings for magnetic resonance imaging of the brain. A high growth velocity ( $>6 \mathrm{~cm} /$ year), advanced bone age (at least 1 year above chronological age), as assessed by the Greulich and Pyle method, and changes in the uterus and ovaries, as assessed by pelvic ultrasound scans, confirmed progressive iCPP. Patients were classified as having familial iCPP if they had at least one first- or second-degree relative with documented CPP or on the basis of self-reported age at menarche for female relatives or age at onset of pubertal changes in male relatives.

\section{Hormone assays}

Serum LH and follicle-stimulating hormone (FSH) were determined in immunochemiluminescence assays (Siemens Healthcare Diagnostics SAS, Saint Denis, France) and serum estradiol $\left(\mathrm{E}_{2}\right)$ concentration was determined in a RIA (EST-US-CT, Cisbio Bioassays, Gif sur Yvette, France). For the GnRH stimulation test, serum LH and FSH were assayed 15 min before and 20, 40, 60, and 90 min after an i.v. injection of $100 \mu \mathrm{g}$ of GnRH. Basal LH values $>0.5 \mathrm{IU} / \mathrm{l}$ and GnRH-stimulated LH values $>5 \mathrm{IU} / \mathrm{l}$ were considered to be within the pubertal range. The intra-assay coefficients of variation (CV) values were $4 \%$ for $\mathrm{FSH}, 3.2 \%$ for $\mathrm{LH}$, and $5 \%$ for $\mathrm{E}_{2}$. The inter-assay $\mathrm{CV}$ values were $5 \%$ for $\mathrm{FSH}, 7 \%$ for $\mathrm{LH}$, and $10 \%$ for $\mathrm{E}_{2}$.

\section{Pelvic ultrasound scans}

Pelvic ultrasound examinations were carried out at the time of diagnosis of precocious puberty. The following signs of estrogen exposure were sought: uterine length $>34 \mathrm{~mm}$, pear-shaped uterus, and endometrial thickening.

\section{Genetic testing}

DNA was collected from the index cases, their parents, and other affected family members, when possible. Written informed consent for genetic testing was obtained from the parents of each index patient and from all the family members tested. This retrospective study has been approved by the Ethical Committee of Robert Debré Hospital (\#2014-158). Genomic DNA was extracted from peripheral blood lymphocytes. The screening of KISS1 and KISS1R as described elsewhere $(9,17)$ identified no mutations. The MKRN3 coding region was amplified with two pairs of primers described by Abreu et al. (11) and an additional pair of primers (5'-GATTGGCTCGGCTGCTGAAAG-3' and 5'-TGGGCAAGACTTGACGATCCT-3'). The pathogenicity of the variants was assessed with the in silico prediction programs SIFT and Mutation Taster, and we checked that the variants identified were absent from two databases (1000 Genomes and NHLBI EVS). 
Table 1 Primers used to quantify MKRN3 alleles.

\begin{tabular}{l} 
Names \\
\hline MKRN3-1 \\
MKRN3-2 \\
MKRN3-3 \\
RB1 \\
MYH9
\end{tabular}

\begin{tabular}{l} 
Forward \\
\hline CAAAGCAGCCATGGAAGA \\
ATTATGCTTCCAGGGGAGT \\
GCGGTTTCTTTCACTGAGA \\
GATCTCCAAAGAAAAAAGGTCAACTACG \\
CTCCCCGCTTAGGTCCTGGCTAT
\end{tabular}

Reverse

GCTCACAGACGGGAAGGT

ATGTTCTTCCCTCTGGGC

CTAGACCACATGCCACAGC

GGTAGATTTCAATGGCTTCTGGGTCTG GGCCAGCGGATTGTTGATGAAG
As iCPP has been reported in a few patients with Prader-Willi syndrome (16), we hypothesized that a deletion restricted to MKRN3 might be associated with iCPP in patients without the Prader-Willi phenotype. We developed a quantitative PCR gene dosage assay for MKRN3, involving three primer pairs (Table 1). We used PCR to amplify $25 \mathrm{ng}$ of genomic DNA in $20 \mu \mathrm{l}$ of PCR mix (Applied Biosystems), with each primer at a concentration of $500 \mathrm{nM}$. RB1 and MYH9 were used as reference genes for $M K R N 3$ quantification. All reactions were performed in triplicate. DNA from a patient with Prader-Willi syndrome and a deletion encompassing $M K R N 3$ was used as a positive control and omission of the DNA template as a negative control. PCR conditions were as follows: $10 \mathrm{~min}$ of denaturation at $95^{\circ} \mathrm{C}$, followed by 40 cycles of $95^{\circ} \mathrm{C}$ for $15 \mathrm{~s}$ and $60^{\circ} \mathrm{C}$ for $60 \mathrm{~s}$, in a 9700 PCR machine (Applied Biosystems). Raw data were then exported to SDS Software for analysis (Applied Biosystems). We determined gene copy number by the $2^{-\Delta \Delta C T}$ method, in which the cycle threshold $(C T)$ is the number of cycles required to reach the fluorescence threshold, $\Delta C \mathrm{~T}$ is the difference in $C \mathrm{~T}$ values between MKRN3 and the reference gene, and $\Delta \Delta C \mathrm{~T}$ is the subtraction of the control $\Delta C$ T from the patient $\Delta C \mathrm{~T}$. The efficiency of quantitative PCR was evaluated by testing a series of DNA dilutions, from 40 to $2 \mathrm{ng} /$ reaction.

\section{Statistical analysis}

Data are reported as medians (interquartile range). Clinical, hormonal, and radiological data were compared between patients with and without MKRN3 mutations, in the nonparametric Mann-Whitney test. $P$ values $<0.05$ were considered statistically significant.

\section{Results}

\section{Patients}

In total, 46 patients were included, 28 of whom had one or more affected relatives (multiplex families), the other 18 being considered to be sporadic cases. Sixteen of the 28 multiplex families contained two iCPP cases, eight included three iCPP cases and four families had four iCPP cases (Fig. 1 and Supplementary Figure 1, see section on supplementary data given at the end of this article). A history of iCPP was reported in the mothers of the index cases in 13 families and in the father of the index case in only one family. In 17 families, iCPP was observed in at least one sibling of the index case.

\section{Mutations}

DNA samples were available for the 28 index cases from the multiplex families, the 18 sporadic cases and for 37 of
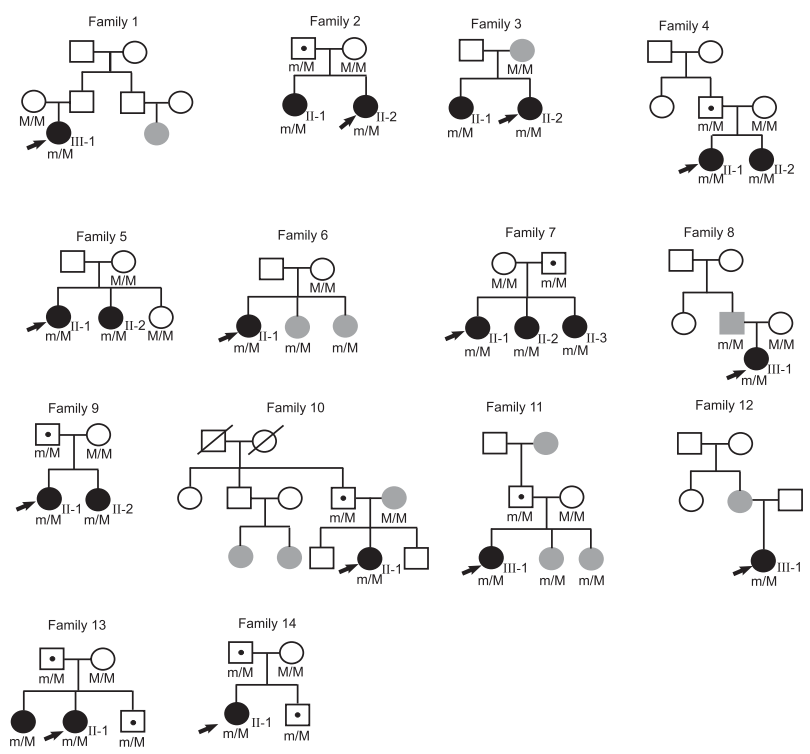

\section{Figure 1}

Pedigrees of patients with MKRN3 mutations. Arrow, proband; open square, male; open circle, female; black symbols, clinically affected subjects; gray symbols, diagnosis on the basis of selfreported age at onset of puberty; black dot in an open square, asymptomatic carriers; m, mutated allele; M, WT allele. Roman numerals indicate the generation. Arabic numerals indicate the patients in each nuclear family. A line crossing the symbol indicates that the patient is deceased. 
their first-degree relatives (mothers, $n=13$; fathers, $n=9$; sisters, $n=13$; and brothers, $n=2$ ). Two frameshift mutations and five heterozygous missense mutations of MKRN3 were identified in 13 familial cases and one sporadic case (Fig. 2). One of the two frameshift mutations identified here has been reported before (c.482insC; p.Ala162Glyfs*15) (11). We found this mutation in eight families (nos 1, 2, 6, 7, 9, 10, 11, and 13). The other frameshift mutation (c.802-803del; p.Met268Valfs*23), found in family no. 12, has never been described before. In silico analysis predicted that these two frameshift mutations would result in the production of truncated proteins of 176 and 290 amino acids respectively. We also identified four missense mutations, c.943A $>\mathrm{G}$; p.Met315Val, c.982C $>$ T; p.Arg328Cys, c.1118C $>$ T; p.Pro373Leu, and c89C $>\mathrm{T}$; p.Pro30Leu, in the index cases of family nos $3,4,5$, and 8 respectively (Fig. 2). The patient identified as a sporadic case was found to have a missense mutation (c.737A $>$ G; p.Tyr246Cys). These five missense mutations were not present in 1000 Genomes and NHLBI EVS databases. The missense mutations identified affected the second zinc finger domain (p.Tyr 246Cys), the C3HC4 RING finger motif (p.Met315Val and p.Arg328Cys), the N-terminus of the protein (p.Pro30Leu) or the region between the C3HC4 RING finger motif and the third zinc finger domain (p.Pro373Leu) (Fig. 2). In silico analysis with two programs predicted that all the missense mutations would result in a loss of function. We investigated the possibility of patients without identified MKRN3 mutations having partial or complete MKRN3 deletion, by determining the number of MKRN3 alleles by quantitative PCR. All patients without
MKRN3 mutations were found to have two alleles of MKRN3.

Paternal inheritance of the mutations was established in nine families, including the sporadic case. Eight of the nine fathers had no symptoms. The remaining father had a history of precocious puberty (family no. 8; Fig. 1). Paternal DNA was not available for five families. In four of these families (nos 1, 3, 5, and 6), no mutation was detected in the mothers, suggesting that the mutations found in the index cases were transmitted by the father. DNA was not available for either of the patients from family no. 12 or for four of the affected relatives of index cases. Interestingly, there was a deficit of boys among the siblings of index cases $(4 / 20)$.

\section{Clinical phenotypes in girls with and without MKRN3 mutations}

MKRN3 mutations were found in 25 iCPP patients from multiplex families and in one sporadic case. Complete clinical data were available for 20 cases from these 13 families (13 index cases and seven relatives) and for the sporadic case $(n=21)$ (Table 2$)$. All these patients were girls. Median age at puberty onset was 6 years (5.4-6) and median age at first evaluation was 6.5 years (5.9-7.3). At this age, these patients displayed the typical features of precocious puberty, including Tanner stage 3, a high height velocity, and advanced bone age (Table 2 ).

Phenotypic data were collected for 34 iCPP girls without MKRN3 mutations, including 15 index cases from 15 families plus three relatives, and 16 sporadic cases. The male patient with sporadic iCPP was excluded

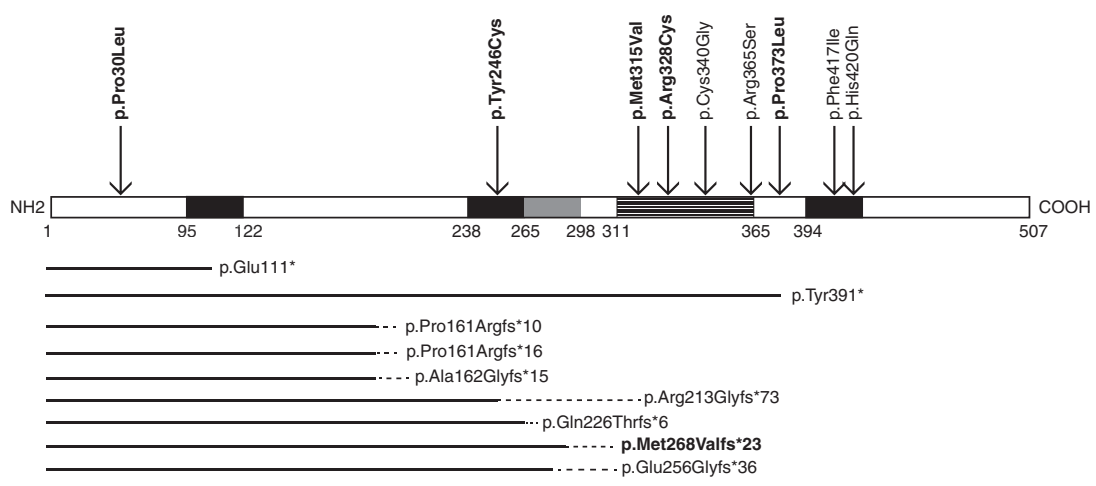

Figure 2

Location of the MKRN3 mutations identified in patients with idiopathic central precocious puberty. Black boxes, zinc finger domains; gray box, MKRN-type motif; hatched box, C3HC4 RING finger motif. Amino-acid residues are indicated. Bold type, mutations reported in this study. 
Table 2 Clinical data for 21 girls (14 index cases + seven siblings) with MKRN3 mutations.

\begin{tabular}{|c|c|c|c|c|c|c|c|c|c|c|c|c|c|c|}
\hline \multirow[b]{2}{*}{ Family } & \multirow[b]{2}{*}{ n } & \multicolumn{8}{|c|}{ Initial diagnosis: clinical and radiological data } & \multicolumn{5}{|c|}{ GnRH stimulation test } \\
\hline & & $\begin{array}{l}\text { Age at } \\
\text { onset }\end{array}$ & $\begin{array}{l}\text { Age at } \\
\text { referral }\end{array}$ & $\begin{array}{l}\text { Tanner } \\
\text { stage } \\
\text { (B) }\end{array}$ & $\begin{array}{l}\text { Height } \\
\text { SDS }\end{array}$ & $\begin{array}{l}\text { Height } \\
\text { velocity } \\
\text { SDS }\end{array}$ & $\begin{array}{l}\text { BMI } \\
\text { SDS }\end{array}$ & $\begin{array}{c}\text { BA } \\
\text { (years) }\end{array}$ & $\begin{array}{l}\text { Uterus } \\
\text { length } \\
(\mathrm{mm})\end{array}$ & $\begin{array}{c}\text { FSH } \\
\text { (IU/l), } \\
\text { basal }\end{array}$ & $\begin{array}{c}\mathrm{FSH}(\mathrm{IU} / \mathrm{l}), \\
\text { peak }\end{array}$ & $\begin{array}{l}\text { LH } \\
\text { (IU/I), } \\
\text { basal }\end{array}$ & $\begin{array}{c}\text { LH }(\mathrm{IU} / \mathrm{I}), \\
\text { peak }\end{array}$ & $\begin{array}{c}E_{2} \\
(p g / m l)\end{array}$ \\
\hline 1 & III-1 & 6.0 & 6.5 & 3 & 3.9 & 2.6 & 0.9 & 9.5 & 46 & 4 & 17.2 & 0.5 & 30.8 & 33 \\
\hline \multirow[t]{2}{*}{2} & II-1 & 6.5 & 8.0 & 4 & 1.4 & 1.7 & 1.6 & 11 & 60 & 5.5 & 13.7 & 2.7 & 36.8 & 25 \\
\hline & II-2 & 5.5 & 5.8 & 2 & 0.7 & NA & -0.02 & 7 & 36 & 1.4 & 18.2 & 0.1 & 25.4 & 37 \\
\hline \multirow[t]{2}{*}{3} & II-1 & 6.0 & 7.6 & 2 & 1.9 & 3 & 1.3 & 11 & 39 & 7.3 & 11.1 & 3.7 & 30.6 & 57 \\
\hline & II-2 & 5.5 & 6.8 & 4 & 0.2 & 1.4 & 0.4 & 7 & 38 & 5.6 & 10 & 1.9 & 31.5 & 10 \\
\hline \multirow[t]{2}{*}{4} & II-1 & 6.0 & 7.8 & 3 & 1.1 & 4.2 & -0.3 & 10.5 & 46 & 5.1 & 11.5 & 2.3 & 28 & 20 \\
\hline & II-2 & 7.0 & 7.8 & 3 & 1.9 & 4.2 & -0.5 & 10.5 & 37 & 2.1 & 14 & 1.1 & 27 & 2 \\
\hline \multirow[t]{2}{*}{5} & II-1 & 3.5 & 3.6 & 2 & 5.6 & 5.8 & 2.8 & 6.8 & 44 & 4.1 & $N D$ & 1.4 & ND & 7 \\
\hline & II-2 & 5.8 & 6.3 & 3 & 4.7 & 5.6 & -0.05 & 8.8 & 35 & 4.9 & 12 & 0.3 & 6.4 & 28 \\
\hline 6 & II-1 & 6.0 & 6.1 & 2 & 2.6 & 1.1 & 0.7 & 8.8 & 43 & 2.5 & 13.2 & 0.3 & 4.9 & NA \\
\hline \multirow[t]{3}{*}{7} & II-1 & 6.0 & 6.7 & 3 & 1.5 & 2.6 & 1.2 & 9 & 32 & 3.7 & 19.8 & 1 & 27.4 & 24 \\
\hline & II-2 & 6.0 & 8.4 & 2 & 1.0 & 2.2 & 1.5 & 8.4 & 49 & 1.9 & 12.4 & 0.1 & 14.4 & 12 \\
\hline & II-3 & 5.6 & 5.7 & 3 & 1.1 & 2.6 & 2.0 & 7.7 & 41 & 2.8 & 31.5 & 0.7 & 22.2 & 12 \\
\hline 8 & III-1 & 5.0 & 7.3 & 3 & 2.6 & 1.6 & 1 & 11 & 40 & 3.2 & 18 & 0.5 & 28.6 & 9 \\
\hline \multirow[t]{2}{*}{9} & II-1 & 4.8 & 6.1 & 3 & 1.3 & 4.4 & 0.7 & 7.8 & 48 & 3.7 & 17.5 & 1.1 & 26.6 & NA \\
\hline & II-2 & 6.8 & 7.0 & 3 & 0.02 & 2.9 & 1.9 & 8.5 & 38 & 9.2 & 11.3 & 0.4 & 20 & NA \\
\hline 10 & III-1 & 5.4 & 5.9 & 2 & 0.2 & NA & 0.9 & 5 & 28 & 6.2 & 12.5 & 1.3 & 18.5 & 19 \\
\hline 11 & III-1 & 6.3 & 6.6 & 3 & 2.2 & 0.9 & 2.6 & 8.5 & 42 & 0.7 & 9 & 0.7 & 12.9 & 34 \\
\hline 12 & III-1 & 3.5 & 3.8 & 3 & 1.6 & 1.6 & 1 & 3.5 & 50 & 3.9 & 16.8 & 0.4 & 33 & 21 \\
\hline 13 & II-1 & 5.0 & 5.8 & 2 & 1.4 & 0.1 & 2 & 6.5 & 32 & 3 & 14 & 0.4 & 9 & 5 \\
\hline 14 & II-1 & 7.5 & 8.3 & 3 & 1.1 & NA & 0.08 & 11 & 47 & 4.3 & 15.5 & 1.1 & 27.6 & $<5$ \\
\hline Median & & 6.0 & 6.5 & 3 & 1.4 & 2.4 & 0.09 & 8.5 & 41 & 3.9 & 13.8 & 0.7 & 26.8 & 19 \\
\hline Q1-Q3 & & $5.4-6$ & $5.9-7.3$ & $2-3$ & $1.1-2.2$ & $1.6-4.2$ & $0.4-1.9$ & $7-10.5$ & $37-46$ & $2.8-5.1$ & $11.7-17.3$ & $0.4-1.3$ & $16.4-29.6$ & $9-28$ \\
\hline
\end{tabular}

$\mathrm{BA}$, bone age; $\mathrm{LH}$, luteinizing hormone; $\mathrm{FSH}$, follicle-stimulating hormone; $\mathrm{E}_{2}$, estradiol; ND, not done; NA, not available. Normal prepubertal values are as follows: $\mathrm{E}_{2}<10 \mathrm{pg} / \mathrm{ml} ; \mathrm{LH}<0.5 \mathrm{IU} / \mathrm{l} ;$ and post-GnRH peak $<5 \mathrm{IU} / \mathrm{l}$.

from this analysis. Girls with MKRN3 mutations were significantly younger at puberty onset than those without such mutations (6.0 years (5.4-6) vs 7.0 years (6-7), $P=0.01)$. They were also significantly younger at the first evaluation (6.5 years (5.9-7.3) vs 8.0 years (6.9-8.3), $P=0.004)$. No differences in hormone levels or ultrasound evidence of estrogen exposure were found between girls with and without mutations (Table 3).

\section{Discussion}

Thirteen of the 28 familial cases of iCPP had heterozygous MKRN3 mutations identified by genetic screening. Six heterozygous mutations of MKRN3 were found in these familial cases: four new missense mutations and two frameshift mutations, one of which had never before been identified. In addition, a new missense mutation was found in a girl with sporadic iCPP. A comparison of endocrine phenotypes between girls with and without mutations showed that those with MKRN3 mutations were younger at iCPP onset than those without such mutations.
In 2013, Abreu et al. (11) described the first families with iCPP and heterozygous MKRN3 mutations. These mutations were found in 15 patients from five families. Thirteen other patients with iCPP and MKRN3 mutations, from six families, have since been described $(12,13,14,15)$. In our study, heterozygous MKRN3 mutations were found in 14 of 46 index patients with iCPP (30\%). All the mutations identified were predicted to be loss-of-function mutations by in silico analysis methods, and were therefore suspected to be the cause of the precocious puberty. The c.482ins $C$ frameshift mutation has already been reported elsewhere (11). The high frequency of this mutation in our study (8/14) confirms that the cytosine homopolymer between nucleotides 476 and 482 is a mutation hotspot. As expected, the proportion of mutations was high in the subgroup with familial iCPP $(n=13 / 28,46 \%)$. Only one of the 18 girls with sporadic iCPP had an MKRN3 mutation, consistent with findings for a large group of sporadic cases (14).

In the subgroup with familial iCPP, segregation analysis predicted a high probability of the mutation having been transmitted to the affected children by their 
Table 3 Phenotypic comparison between girls with and without MKRN3 mutations. Values are expressed as the median (interquartile range).

\begin{tabular}{lc}
\hline & MKRN3 mutated girls $(n=21)$ \\
\hline Age at puberty onset (years) & $6(5.4-6)$ \\
Age at evaluation (years) & $6.5(5.9-7.3)$ \\
Breast Tanner stage & $3(2-3)$ \\
Height SDS & $1.4(1.1-2.2)$ \\
BMI SDS & $0.9(0.4-1.9)$ \\
BA (years) & $8.5(7-10.5)$ \\
Delta BA-CA (years) & $2.4(1.5-2.7)$ \\
Uterus length (mm) & $41(37-46)$ \\
LH (IU/I) & $0.7(0.4-1.4)$ \\
Basal & $26.8(16.4-29.6)$ \\
Peak & \\
FSH (IU/l) & $3.9(2.8-5.1)$ \\
Basal & $13.8(11.7-17.3)$ \\
Peak & $19(9-28)$ \\
Estradiol (pg/ml) &
\end{tabular}

\begin{tabular}{c}
\hline MKRN3 non-mutated girls $(n=34)$ \\
\hline $7(6-7)$ \\
$8(6.9-8.3)$ \\
$3(2-3)$ \\
$2(1.5-2.7)$ \\
$1.1(0.4-1.4)$ \\
$10.2(8.5-11)$ \\
$2.5(1.6-3.2)$ \\
$45(37-52)$ \\
$1.1(0.5-2.8)$ \\
$12.5(8-41)$ \\
$4(3-5.3)$ \\
$14.8(11.5-21)$ \\
$16(7-31)$
\end{tabular}

\begin{tabular}{ll}
\hline $\boldsymbol{P}$ value \\
\hline 0.01 \\
0.004 \\
0.49 \\
0.15 \\
0.7 \\
0.059 \\
0.41 \\
0.21 \\
0.16 \\
0.27 \\
0.72 \\
0.82 \\
0.94
\end{tabular}

$\mathrm{BA}$, bone age; $\mathrm{CA}$, chronological age; $\mathrm{LH}$, luteinizing hormone; FSH, follicle-stimulating hormone.

fathers. A history of iCPP was reported in the father (family no. 8), in paternal first cousins (family nos 1 and 10), or in the paternal grandmother (family no. 11). These cases were consistent with the need for mutations in a paternally expressed gene, such as MKRN3, to be transmitted by the father to cause the phenotype. However, a history of iCPP in the mother does not exclude the presence of an MKRN3 mutation, as shown by family nos 3 and 10.

The large number of patients with familial iCPP for whom comprehensive data were collected made it possible to analyze the segregation of MKRN3 mutations with iCPP. It also led to the description of new MKRN3 mutations. However, this study also had limitations, due to its observational nature and the use of declarative data for pubertal status. A history of iCPP was reported in only one of the nine fathers carrying MKRN3 mutations. In previously reported familial cases with MKRN3 mutations, the fathers were reported to be asymptomatic carriers in nine of 11 cases, whereas inheritance of the mutation from the paternal grandmother was confirmed in only two cases $(11,15)$. This may reflect the inheritance of the mutation, by fathers, from their mothers, as in family no. 11. This suspected overrepresentation of transmission of the mutated allele from the paternal grandmother raises questions about the veracity of the age at onset of puberty self-reported by fathers with mutations. Indeed, puberty onset is harder to recognize in boys than in girls, and cases of precocious puberty may have been missed. This analysis of large pedigrees with iCPP suggests that screening for MKRN3 mutations should be performed in all familial cases with potential paternal transmission, or at least two affected siblings if there is no paternal history of iCPP.

Thirty-three patients with MKRN3 mutations have been reported to date. More girls $(n=23)$ than boys ( $n=10$, eight index cases and two affected relatives) have been found to have such mutations. This is consistent with the findings of our study, in which mutations were identified mostly in female subjects with iCPP. This may reflect a recruitment bias and the well-known higher prevalence of precocious puberty in girls than in boys. No accurate information about age at puberty onset was available for three previously reported cases of iCPP in male patients with MKRN3 mutations $(11,12,13)$. The other five boys had a median age at Tanner stage 2 of 8.25 years (8-8.5), suggesting that the decrease in age at puberty onset was smaller for boys than for girls. Additional studies are required to determine whether the susceptibility to CPP conferred by MKRN3 mutations differs between girls and boys. Clinical follow-up of the two boys with MKRN3 mutations in family nos 13 and 14 should make it possible to evaluate the degree to which MKRN3 mutations are expressed in boys.

MKRN3 is located on chromosome 15. We would therefore expect there to be similar numbers of boys and girls among the offspring of men with MKRN3 mutations. However, we actually observed a deficit of boys in these 14 families (Fig. 1), and these findings are consistent with published data. Indeed, the offspring of the 15 previously reported fathers with MKRN3 mutations (11, 12, 13, 14, 15) consists of 25 girls and six boys, consistent with the imbalance in the numbers of boys and girls observed in 
our families. This deficit of boys among the offspring of mutated fathers requires confirmation in a larger group of men with mutations, but it suggests that Y-bearing sperm cells carrying MKRN3 mutations may be defective.

We compared phenotypes in girls with and without MKRN3 mutations, to determine whether, in addition to familial history, there were any clinical or biological features suggestive of MKRN3 mutation. Median age at puberty onset was the only parameter found to differ significantly between girls with and without mutations (lower in girls with mutations, at 6.0 years (5.4-6)) Similarly, the median age at puberty onset in 22 previously reported cases in girls was 6.0 years $(5.4-6.1)(11,12,13$, $14,15)$. The narrow range of age at puberty onset in patients with MKRN3 mutations indicates that the activity of the encoded protein is critical at a specific time point in the postnatal development of the GnRH neuronal network. We identified no differences in hormonal status between girls with and without MKRN3 mutations, although higher FSH levels were recorded in the subgroup of girls with mutations in a study of a large group of patients with sporadic CPP described elsewhere (14). In their cohort of sporadic cases of iCPP, Macedo et al. (14) found no difference in age at puberty onset between patients with and without MKRN3 mutations. Moreover, preliminary results from our group indicate that the median age at puberty of index cases does not seem to be different between patients with a maternal transmission of CPP as compared to those with a paternal transmission (Our group, personal communication). The differences in findings between these two studies may be due to the diverse causes of iCPP familial cases as compared to sporadic cases.

The mechanism by which MKRN3 mutations affect the onset of puberty is unknown. It has been suggested that the MKRN3 protein inhibits GnRH secretion in mice (11). A recent study on a large group of healthy Danish girls showed that serum MKRN3 concentration declined before the onset of puberty and was lower in a subset of girls with early puberty onset than in the subset of age-matched prepubertal girls (18). A recent genome wide-association study identified two single-nucleotide polymorphisms of MKRN3 significantly associated with an older age at menarche only when inherited from the father (19). These SNPs were not associated with serum MKRN3 concentration (18), but they might be associated with a persistence of intracellular MKRN3 activity for a longer period of time after birth than for the WT allele (18).

All the children with MKRN3 mutations in our study have been or are still being treated with GnRH agonists and have shown a good treatment response, consistent with other reports $(11,14)$. Menarche occurred normally after the cessation of treatment. These data suggest that MKRN3 may be involved in controlling gonadotropic axis reactivation during childhood but with no effect on the GnRH-induced LH surge leading to ovulation in females at the end of the follicular phase (20). The timing of gonadotropic axis reactivation differs between boys and girls (21). This difference in timing between the sexes persists in patients with MKRN3 mutations. Rather than controlling GnRH secretion directly, MKRN3 may modulate postnatal gonadotropic axis maturation in both sexes.

In conclusion, the identification of MKRN3 mutations in patients with iCPP has constituted a major breakthrough in the understanding of iCPP genetics. The prevalence of MKNR3 mutations is high in familial cases of iCPP. The onset of iCPP occurs earlier in patients with MKRN3 mutations than in those without such mutations, and sexual dimorphism for age at puberty onset persists in patients with mutations. These MKRN3 mutations accelerate the postnatal development of the gonadotropic axis. Additional genetic studies are required to identify genetic abnormalities associated with maternally transmitted iCPP.

\section{Supplementary data}

This is linked to the online version of the paper at http://dx.doi.org/10.1530/ EJE-15-0488.

\section{Declaration of interest}

The authors declare that there is no conflict of interest that could be perceived as prejudicing the impartiality of the research reported.

\section{Funding}

This study was supported by INSERM and DHU PROTECT.

\section{Acknowledgements}

The authors are grateful to the patients and their families, and to the pediatricians who collected the clinical data for the index cases.

\section{References}

1 Carel JC \& Leger J. Clinical practice. Precocious puberty. New England Journal of Medicine 2008358 2366-2377. (doi:10.1056/ NEJMcp0800459)

2 Chalumeau M, Hadjiathanasiou CG, Ng SM, Cassio A, Mul D, Cisternino M, Partsch CJ, Theodoridis C, Didi M, Cacciari E et al. Selecting girls with precocious puberty for brain imaging: validation of 
European evidence-based diagnosis rule. Journal of Pediatrics 2003143 445-450. (doi:10.1067/S0022-3476(03)00328-7)

3 Tomkins DJ, Roux AF, Waye J, Freeman VC, Cox DW \& Whelan DT. Maternal uniparental isodisomy of human chromosome 14 associated with a paternal $\mathrm{t}(13 \mathrm{q} 14 \mathrm{q})$ and precocious puberty. European Journal of Human Genetics 19964 153-159.

4 Mutesa L, Hellin AC, Jamar M, Pierquin G, Bours V \& Verloes A. Precocious puberty associated with partial trisomy $18 \mathrm{q}$ and monosomy 11q. Genetic Counseling 200718 201-207.

5 Partsch CJ, Japing I, Siebert R, Gosch A, Wessel A, Sippell WG \& Pankau R. Central precocious puberty in girls with Williams syndrome. Journal of Pediatrics 2002141 441-444. (doi:10.1067/mpd.2002.127280)

6 de Vries L, Kauschansky A, Shohat M \& Phillip M. Familial central precocious puberty suggests autosomal dominant inheritance. Journal of Clinical Endocrinology and Metabolism 200489 1794-1800. (doi:10.1210/jc.2003-030361)

7 Silveira LG, Noel SD, Silveira-Neto AP, Abreu AP, Brito VN, Santos MG, Bianco SD, Kuohung W, Xu S, Gryngarten M et al. Mutations of the KISS1 gene in disorders of puberty. Journal of Clinical Endocrinology and Metabolism 201095 2276-2280. (doi:10.1210/jc.2009-2421)

8 Teles MG, Bianco SD, Brito VN, Trarbach EB, Kuohung W, Xu S, Seminara SB, Mendonca BB, Kaiser UB \& Latronico AC. A GPR54activating mutation in a patient with central precocious puberty. New England Journal of Medicine 2008358 709-715. (doi:10.1056/ NEJMoa073443)

9 Huijbregts L, Roze C, Bonafe G, Houang M, Le Bouc Y, Carel JC, Leger J, Alberti P \& de Roux N. DNA polymorphisms of the KiSS1 3' untranslated region interfere with the folding of a G-rich sequence into G-quadruplex. Molecular and Cellular Endocrinology 2012351 239-248. (doi:10.1016/j.mce.2011.12.014)

10 Leka-Emiri S, Louizou E, Kambouris M, Chrousos G, De Roux N \& Kanaka-Gantenbein C. Absence of GPR54 and TACR3 mutations in sporadic cases of idiopathic central precocious puberty. Hormone Research in Paediatrics 201481 177-181. (doi:10.1159/000356913)

11 Abreu AP, Dauber A, Macedo DB, Noel SD, Brito VN, Gill JC, Cukier P, Thompson IR, Navarro VM, Gagliardi PC et al. Central precocious puberty caused by mutations in the imprinted gene MKRN3. New England Journal of Medicine 2013368 2467-2475. (doi:10.1056/ NEJMoa1302160)

12 Settas N, Dacou-Voutetakis C, Karantza M, Kanaka-Gantenbein C, Chrousos GP \& Voutetakis A. Central precocious puberty in a girl and early puberty in her brother caused by a novel mutation in the MKRN3 gene. Journal of Clinical Endocrinology and Metabolism 201499 E647-E651. (doi:10.1210/jc.2013-4084)

13 Schreiner F, Gohlke B, Hamm M, Korsch E \& Woelfle J. MKRN3 mutations in familial central precocious puberty. Hormone Research in Paediatrics 201482 122-126. (doi:10.1159/000362815)

14 Macedo DB, Abreu AP, Reis AC, Montenegro LR, Dauber A, Beneduzzi D, Cukier P, Silveira LF, Teles MG, Carroll RS et al. Central precocious puberty that appears to be sporadic caused by paternally inherited mutations in the imprinted gene makorin RING finger 3. Journal of Clinical Endocrinology and Metabolism 201499 E1097-E1103. (doi:10.1210/jc.2013-3126)

15 de Vries L, Gat-Yablonski G, Dror N, Singer A \& Phillip M. A novel MKRN3 missense mutation causing familial precocious puberty. Human Reproduction 201429 2838-2843. (doi:10.1093/humrep/ deu256)

16 Crino A, Di Giorgio G, Schiaffini R, Fierabracci A, Spera S, Maggioni A \& Gattinara GC. Central precocious puberty and growth hormone deficiency in a boy with Prader-Willi syndrome. European Journal of Pediatrics 2008167 1455-1458. (doi:10.1007/s00431-0080679-0)

17 de Roux N, Genin E, Carel JC, Matsuda F, Chaussain JL \& Milgrom E. Hypogonadotropic hypogonadism due to loss of function of the KiSS1-derived peptide receptor GPR54. PNAS 2003100 10972-10976. (doi:10.1073/pnas.1834399100)

18 Hagen CP, Sorensen K, Mieritz MG, Johannsen TH, Almstrup K \& Juul A. Circulating MKRN3 levels decline prior to pubertal onset and through puberty: a longitudinal study of healthy girls. Journal of Clinical Endocrinology and Metabolism 2015100 1920-1926. (doi:10.1210/ jc.2014-4462)

19 Perry JR, Day F, Elks CE, Sulem P, Thompson DJ, Ferreira T, He C, Chasman DI, Esko T, Thorleifsson G et al. Parent-of-origin-specific allelic associations among 106 genomic loci for age at menarche. Nature 2014514 92-97. (doi:10.1038/nature13545)

20 Herbison AE. Physiology of the adult gonadotropin-releasing hormone neuronal network. In Physiology of Reproduction, ch 28. pp 399-467. Eds TM Plant \& AJ Zeleznik. London: Elsevier Academic Press, 2015.

21 Ojeda SR \& Lomniczi A. Puberty in 2013: unravelling the mystery of puberty. Nature Reviews. Endocrinology 201410 67-69. (doi:10.1038/ nrendo.2013.233)

Received 13 May 2015

Revised version received 10 September 2015

Accepted 1 October 2015 